\title{
Conocimiento emocional de profesores de matemáticas
}

\author{
Emotional knowledge of mathematics teachers
}

\author{
María S. García-González ${ }^{1}$ \\ Oswaldo Jesús Martínez-Padrón²
}

Resumen: Se presenta una reflexión sobre experiencias educativas en torno al conocimiento emocional del profesorado de matemáticas, en el marco de nuestra interacción con docentes en talleres de matemática emocional y clases de un posgrado en Docencia de la Matemática, en México. Desde dicha experiencia, se proponen dos técnicas que pueden ayudar al docente a desarrollar su conocimiento emocional.

Palabras Clave: Conocimiento emocional, profesores, matemáticas.

\begin{abstract}
In this article we provide a reflection about educational experiences around the emotional knowledge of mathematics teachers, within the framework of our interaction with teachers in emotional mathematics workshops and postgraduate classes in Mathematics Teaching, in Mexico. From this experience, we propose two techniques that can help teachers to develop their emotional knowledge.
\end{abstract}

Keywords: Emotional knowledge, teachers, mathematics.

Fecha de recepción: 17 de noviembre de 2018. Fecha de aceptación: 26 de noviembre de 2019

${ }^{1}$ Facultad de Matemáticas. Universidad Autónoma de Guerrero, México; mgargonza@gmail.com; https:// orcid.org/0000-0001-7088-1075.

2 Universidad Pedagógica Experimental Libertador (Venezuela)- Universidad Técnica del Norte (Ecuador); Facultad de Ciencias Administrativas y Económicas; ommadail@gmail.com; https://orcid.org/0000-0002-4142-8092 


\section{EL AFECTO EN LA ENSEÑANZA DE LAS MATEMÁTICAS}

"Para que una cosa sea útil, hay que usarla, pero lo que se odia, más se maltrata que se usa" (Mager, 1984, p. 1). Con esta frase, Robert Mager, uno de los nombres más reconocido en el campo de la formación y la mejora del rendimiento educativo americano, inicia su libro Developing attitude toward learning dirigido a profesores y personas interesadas en la educación, título que se corresponde con el objetivo de la obra, ya que trata de principios que los profesores pueden usar para influir positivamente en la actitud de los estudiantes, con la finalidad de que estos terminen su educación no solo con deseos de emplear lo aprendido sino ávidos de aprender más. Reflexiones como la anterior nos llevan a reafirmar la importancia que se le ha otorgado a los factores afectivos en el proceso de enseñanza-aprendizaje en cualquier campo del saber.

En cuanto a las matemáticas, como asignatura escolar, el afecto también ha sido motivo de preocupación de expertos desde hace décadas, basta mencionar al matemático húngaro George Polya quien, desde 1945, ya había advertido que las emociones juegan un papel preponderante en la resolución de problemas matemáticos, haciendo mención a cuestiones asociadas con la voluntad y la determinación, la cual varía según la esperanza o el abatimiento y la satisfacción o la desilusión (Polya, 1965). Casi cuatro décadas más tarde, Schoenfeld (1983), educador matemático, hizo hincapié en el papel que juega el sistema de creencias en la determinación de la toma de decisión de un estudiante mientras resuelve problemas matemáticos, apuntando que las actitudes hacia las matemáticas y la confianza en ellas pueden ser los aspectos que influyen en cómo los estudiantes manejan sus recursos cognitivos.

Esta importancia del afecto en la enseñanza-aprendizaje de las matemáticas llevó al educador matemático Douglas McLeod a conceptualizar el Dominio Afectivo como "un extenso rango de sentimientos y humores (estados de ánimo), que son generalmente considerados como algo diferente de la pura cognición, e incluye como componentes específicos de este dominio las actitudes, creencias y emociones" (McLeod, 1989, p. 245). A partir del trabajo de McLeod, la investigación sobre el afecto y el aprendizaje de las matemáticas creció, incorporándose nuevos constructos como los valores (De Bellis y Goldin, 2006), la motivación, la identidad y la autoeficacia (Goldin, et al, 2016), en conjunción con el papel que juegan los estudiantes, los profesores, el saber matemático, la escuela, el discurso y el contexto social correspondiente (Ursini y Sánchez, 2008; Di Martino y Zan, 2010; 2011; Martínez-Padrón, 2016). 
Dentro de ese compendio de actores, instancias y otros aspectos concomitantes, en este artículo centraremos la atención en el profesor de matemáticas y sus emociones, porque reconocemos al salón de clases como una micro-cultura que norma sus comportamientos y su sentir, sin excluir una buena gestión y manejo de las relaciones interpersonales que, junto con la comprensión de conceptos, constituyen la base de la inteligencia social requerida para aprender a vivir en un mundo donde se deben dar muestras, de autenticidad, sintonía, aptitud social, sincronía y empatía (Goleman, 2006; Albrecht, 2006). De acuerdo con Rodríguez, Guevara y Viramontes (2017), en México no se le ha dado la importancia justa al problema de estrés laboral en docentes. Igual ocurre en países como Paraguay y Venezuela (Martínez-Padrón; 2008; 2016), donde muchos profesores de matemáticas son colocados en situaciones tensas que no resultan fáciles de gerenciar, debido a que tienen que atender estudiantes cargados con actitudes desfavorables hacia el estudio.

En otros contextos iberoamericanos, Font (2011) señala que las demandas de los sistemas educativos actuales generan malestar, preocupación y angustia en los docentes a quienes les cuesta concretar las herramientas adecuadas para organizar los contenidos matemáticos que deben enseñar, sobre todo cuando se les cuestiona la manera de enseñarlos en el aula. En el caso de España, Gómez, Blanco, Cárdenas y Guerrero (2012) destacan que quienes enseñan matemáticas se sienten estresados por muchas variables, como la falta de interés, la ausencia de motivación y la indisciplina.

En resumen, en el contexto escolar abundan variadas situaciones cargadas de falta de motivación, angustia, preocupación, consternación, ansiedad y otros factores que impactan notablemente tanto a los protagonistas de la clase como a otros actores e instancias del entorno inmediato. Consideramos que todos estos aspectos deben ser atendidos por estar ligados con el éxito o el fracaso de los estudiantes, sus docentes, sus escuelas y otros elementos concomitantes.

\section{¿QUÉ ES UNA EMOCIÓN?}

Solomon (2008) señala que definir "emoción" es una tarea tan difícil como sería dominarlas, y las considera más peligrosas y comprometidas que la propia razón de los sujetos, aunque esta última es quien debe controlarlas. Agrega que las emociones suelen afectar hasta el juicio de los sujetos implicados, pudiendo estar 
vinculadas con deseos e involucradas con actitudes, creencias, percepciones, estados de ánimo y otros factores morales, sociales, culturales y psicológicos. Stets y Turner (2008) agregan que si la mirada es realizada desde el ámbito sociológico ha de tomarse en cuenta la cultura, en vista de la necesidad de recaudar detalles sobre las regulaciones de los comportamientos de los sujetos mediante sus interacciones comunicacionales. Por nuestra parte nos ceñimos al paradigma cognitivo, desde las teorías de la valoración en psicología, quienes suponen que las emociones son consecuencias de nuestras evaluaciones de ciertos eventos, particularmente nos ceñimos a la Teoría de la Estructura Cognitiva de las Emociones (Ortony, Clore y Collins, 1996), Ilamada comúnmente teoría OCC.

La teoría OCC está fundamentada en la idea de que las emociones son desencadenadas por las valoraciones cognitivas que la gente hace de una situación de manera consciente o no. Desde esta perspectiva, las emociones son entendidas como "reacciones con valencia ante acontecimientos, agentes u objetos, la naturaleza particular de las cuales viene determinada por la manera como es interpretada la situación desencadenante" (Ortony, Clore y Collins, 1996, p. 16), siendo su fuente de evidencia el lenguaje, desde donde es posible conocer los orígenes y las emociones experimentadas por las personas. Con base en este fundamento teórico, para identificar una emoción centramos la atención en la palabra emocional asociada y en la situación que la desencadena.

De acuerdo con lo planteado, se avizora una situación investigativa espinosa sustentada en lo complejo que resulta separar a los elementos constituyentes de las emociones en relación con lo cultural, social, biológico, filosófico o psicológico, sobre todo cuando se hace referencia a componentes de factores como la angustia, la tensión o la depresión producida por el descenso de la autoestima o por la presencia de la desesperanza y el desánimo. Tales aspectos también impactan en la configuración de creencias, actitudes, motivaciones y otros factores del dominio afectivo ligados con el éxito o con el fracaso de los protagonistas de la clase de Matemática (Martínez-Padrón, 2016). Empero, existen senderos como el visionado por la teoría OCC, donde el conocimiento de las valoraciones cognitivas apertura posibilidades de concretar emociones aliadas a situaciones concretas.

Para cerrar esta sección, es oportuno dilucidar algunos aspectos relacionados con el afecto, los sentimientos y las emociones. Damasio (2009) asevera que "la emoción y las reacciones relacionadas están alineadas con el cuerpo, los sentimientos con la mente" (p. 7), de manera que se puede concretar que "los pensamientos desencadenan emociones... [y] las emociones corporales se transforman en el tipo de pensamientos que denominamos sentimientos o sensaciones" 
(p. 6). Este mismo autor cita a Spinoza quien declara al afecto como una serie de "impulsos, motivaciones, emociones y sentimientos" (p. 7), lo cual resulta prudente para allanar las dificultades que a veces se presentan durante el discurso.

\section{LAS EMOCIONES EN LA ENSEÑANZA DE LAS MATEMÁTICAS}

Las emociones del profesorado se clasifican en dos grupos: negativas y positivas. Las negativas implican experiencias desagradables durante el momento de la enseñanza; entre ellas se citan el estrés, la desmotivación y el síndrome de Burnout, un trastorno emocional provocado por el estrés laboral que conlleva a experimentar ansiedad e incluso depresión (Schutz y Zembylas, 2009; Rodríguez, Guevara y Viramontes, 2017). Cuando este grupo de emociones se presenta en grados intensos pueden conducir hasta el abandono de la profesión (Hannula, Liljedahl, Kaasila y Rösken, 2007). Entre las emociones negativas, la que aparece con más frecuencia en los profesores de matemáticas es la llamada ansiedad matemática (Fennema y Sherman, 1976; Hannula, Liljedahl, Kaasila y Rösken, 2007; Bekdemir, 2010). Las emociones positivas, por el contrario, implican experiencias de placer al momento de conducir una clase, entre ellas se encuentran el entusiasmo, la alegría, la satisfacción y el interés (Di Martino y Sabena, 2011; Anttila, Pjyhältö, Soini y Pietarinen, 2016).

De acuerdo con resultados de investigaciones, existen dos razones por las que se desencadenan las emociones negativas de los docentes que enseñan matemática: (a) las experiencias emocionales experimentadas cuando eran estudiantes: generalmente, quienes tuvieron experiencias negativas con las matemáticas las siguen experimentando cuando se convierten en profesores, conservando la creencia de que las matemáticas son difíciles (Di Martino y Sabena, 2011; Coppola, Di Martino, Pacelli y Sabena, 2012); y (b) el conocimiento de la asignatura: muchos de los docentes que tienen la responsabilidad de enseñar matemáticas no siempre son especialistas en los contenidos que les marca el currículo escolar (Philipp, 2007).

Particularmente, nuestras investigaciones sugieren que las emociones, positivas y negativas, del profesor de matemáticas suelen desencadenarse en función de las metas alcanzadas por sus estudiantes en el salón de clases (García-González y Martínez-Sierra, 2016), por ejemplo, "que los estudiantes aprendan". Mientras que muchas de las emociones que experimentan los estudiantes en la clase de matemáticas son desencadenadas por normas del sistema escolar y valoradas por los estudiantes como metas del salón de clases, entre ellas: participar en 
clases, resolver problemas, y graduarse. Si estas metas son alcanzadas, las emociones que experimentan los estudiantes son positivas, en caso contrario, negativas (Martínez-Sierra y García-González, 2014; 2016; 2017).

Haciendo algunas especificaciones sobre los tipos de emociones en referencia, en la Tabla 1 se muestran ambos grupos y varias situaciones desencadenantes que pueden asociarse con emociones particulares de los profesores de matemáticas y de sus estudiantes. Debe tenerse claro que para concretar cualquiera de ellas se hace necesario ligarlas con las vivencias que tales protagonistas dibujan o narran, por ejemplo, en relación con los contenidos matemáticos que son estudiados, enseñados, aprendidos o evaluados. En el caso de los estudiantes, se ha encontrado que un importante contingente de ellos que, históricamente, se ha angustiado por tener la creencia de que las matemáticas son difíciles, le tienen fobia tanto a la asignatura como a quien la enseña (Martínez-Padrón, 2016), a pesar de que existen docentes que se esfuerzan porque sus estudiantes se interesen por la clase y logren aprenderse los contenidos desarrollados en ella.

Tabla 1. Emociones de profesores y estudiantes en matemáticas.

\begin{tabular}{|c|c|c|c|}
\hline \multicolumn{2}{|r|}{ ESTUDIANTES } & \multicolumn{2}{|c|}{ PROFESORES } \\
\hline Emociones & Situaciones desencadenantes & Emociones & $\begin{array}{c}\text { Situaciones } \\
\text { desencadenantes }\end{array}$ \\
\hline $\begin{array}{l}\text { Satisfacción } \\
\text { Decepción } \\
\text { Miedo } \\
\text { Fobia } \\
\text { Aburrimiento } \\
\text { Interés } \\
\text { Júbilo } \\
\text { Congoja } \\
\text { Orgullo } \\
\text { Reproche } \\
\text { Autoreproche } \\
\text { Gusto } \\
\text { Disgusto }\end{array}$ & $\begin{array}{l}\text { Asistir a clases } \\
\text { Participar en clase } \\
\text { Resolver problemas } \\
\text { Aprobar el examen } \\
\text { Aprobar el curso } \\
\text { Graduarse de bachillerato } \\
\text { Entrar a la universidad } \\
\text { Conseguir un empleo }\end{array}$ & $\begin{array}{l}\text { Reproche } \\
\text { Congoja } \\
\text { Júbilo } \\
\text { Agrado } \\
\text { Ira } \\
\text { Fobia } \\
\text { Orgullo } \\
\text { Gratitud } \\
\text { Decepción } \\
\text { Remordimiento } \\
\text { Gratificación } \\
\text { Autoreproche }\end{array}$ & $\begin{array}{l}\text { Que los estudiantes: } \\
\text { Aprendan } \\
\text { Se interesen en la clase } \\
\text { Participen en la clase } \\
\text { Se gradúen } \\
\text { Cursen una carrera } \\
\text { profesional }\end{array}$ \\
\hline
\end{tabular}

Fuente: Martínez-Sierra y García-González, 2014; 2016, 2017; García-González y Martínez-Sierra, 2016.

También existen investigaciones que reportan que en la clase de matemáticas muchos estudiantes aún dejan comandarse por emociones negativas que 
secuestran hasta su capacidad de razonamiento (Goleman, 1996; 2006; Martínez-Padrón, 2008; 2016; Martínez-Padrón, Contarino y Ávila, 2015; Maaß y Schlöglmann, 2009) no logrando vencer los naturales escollos que hay que arrostrar en cualquier evento que pudiera surgir en el aula de matemáticas o en cualquier otro ambiente donde se estén gestando procesos sistematizados que conduzcan al uso de contenidos matemáticos.

\section{EL CONOCIMIENTO EMOCIONAL DEL PROFESOR DE MATEMÁTICAS}

El término conocimiento emocional del profesor de matemáticas es referido al conocimiento que el profesor debe tener sobre sus emociones y la de sus estudiantes durante la enseñanza de esta disciplina (García-González y Pascual-Martín 2017), por lo que tiene que estar consciente de las emociones que se experimentan en el aula y lo que las desencadena, así como de los procederes para su regularización. Con base en Gaxiola (2005) y López y Valls (2013) argumentamos que tener conocimiento emocional implica las siguientes habilidades:

1. Reconocer que sentimos: las emociones son parte de nuestra naturaleza humana.

2. Reconocer qué sentimos: ser capaces de reconocer qué emoción experimentamos ante determinada situación.

3. Concretar, con claridad, la palabra que representa la emoción que realmente sentimos, por ejemplo, no es lo mismo sentir miedo que pavor, sabiendo que este último da cuenta de una intensidad mayor que el miedo.

4. Reconocer qué situación desencadena eso que sentimos.

5. Distinguir las emociones negativas de las positivas.

6. Regular las emociones que experimentamos, siendo capaces de actuar en consecuencia.

7. Poder ayudar a otros a conocerse emocionalmente, por ejemplo a nuestros estudiantes.

Al trabajar con profesores nos hemos percatado que no todos poseen conocimiento emocional que les permita reconocer y gestionar emociones de manera adecuada. Es común que no siempre puedan ponerle nombre a lo que sienten, y al cuestionarles sobre una emoción que experimentan en la clase de matemáticas, responden con la propia palabra emoción para dar cuenta de estados como 
la alegría o satisfacción por algo o por alguien. Por nuestra parte consideramos que los resultados de conocerse emocionalmente pueden ser ventajosos, dado que facilita la toma de decisiones para actuar o detectar el camino correcto que se desea seguir; además de que las emociones evalúan en forma correcta lo que nos pasa, preparándonos para comprender lo que le pasa a otros (Gaxiola, 2005).

\section{ABORDAJE METODOLÓGICO}

Esta investigación está alineada al paradigma fenomenológico-interpretativo, dado que toma en cuenta la interioridad de los profesores de matemáticas que formaron parte de dos grupos: uno constituido por asistentes a un taller sobre el conocimiento emocional del profesor de matemáticas, realizado en una Universidad de Perú, y otro conformado por los participantes de un curso de posgrado en Docencia de la Matemática, en una Universidad de México. Tal alineación la inscribe en el paradigma cualitativo y para materializar las evidencias se solicitó a dichos profesores que dibujaran o narraran eventos o experiencias emocionales, vivencias y motivaciones ligadas con sus clases de matemáticas, seguidas de sus correspondientes significados contextuales. Todo ello involucró un compendio de historias y relatos que tuvieron que ver con su ser, su sentir y su hacer como docente de matemáticas.

El carácter fenomenológico fue asumido según Martínez-Miguélez (2006), quien destaca que la realidad a abordar en este tipo de estudios no es la externa del sujeto sino la interna y personal, por eso toma en cuenta "una realidad cuya esencia depende del modo en que es vivida y percibida por el sujeto" (p. 137).

El conjunto de aspectos teóricos se concretó con una investigación documental donde se incluye la teoría de la OCC y otros aspectos que permitieron no solo visionar el panorama y concretar los postulados de sustento, sino atesorarlos con apoyo de dos técnicas pautadas para identificar emociones.

\section{TÉCNICAS PARA IDENTIFICAR EMOCIONES}

Enseguida se presentan dos técnicas que hemos implementado para identificar emociones, las cuales son útiles para ponerle nombre a la emoción experimentada e identificar la situación que la desencadena. 


\section{EL DIBUJO}

Desde la Matemática Educativa se han realizado estudios usando dibujos para averiguar las creencias que los estudiantes tienen de las matemáticas y de los matemáticos (Picker y Berry, 2000; Aguilar, Rosas y Romo-Vazquez, 2016; Yazlik y Erdogan, 2017). Henrion (1997) sugiere que las imágenes revelan no solo creencias, sino suposiciones y expectativas subyacentes, pudiendo proporcionar ideas de lo que sentimos. Por esta razón, el dibujo es una técnica que hemos replicado y hemos comprobado su funcionalidad al pedirle a los profesores de diferentes niveles escolares, que realicen un dibujo de una experiencia emocional positiva, y una negativa, de cualquiera de las clases de matemáticas que ellos han vivido durante su experiencia laboral.

La indicación que se les da a los docentes es que realicen, de manera individual, el dibujo de una experiencia emocional sin incluir palabras emocionales, después lo muestran al resto de profesores, quienes identificarán la emoción allí expresada (ver Figura 1). Este ejercicio es muy fructífero, ya que tanto el que dibuja como quienes lo identifican, fortalecen su conocimiento emocional al reconocer en sí mismos y en los demás las emociones que se experimentan. Después de que los observadores hablan de la emoción que ven en el dibujo, quien dibujó debe decir si esa era la emoción que de verdad quería comunicar. La mayoría de las veces hay coincidencias por parte de los observadores. Al final se pide a quien dibujó que escriba la palabra emocional asociada con el dibujo. Este ejercicio ayuda, también, a reconocer la situación que desencadena la emoción.

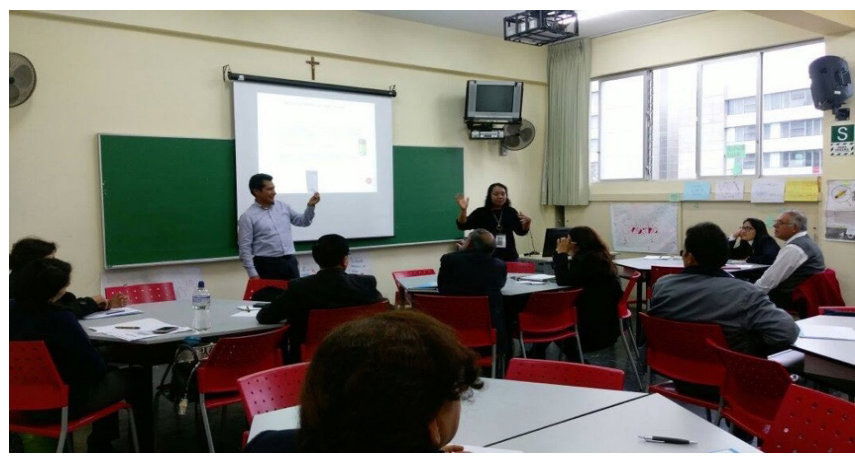

Figura 1. Expresando las emociones mediante dibujos.

Fuente: Taller "El conocimiento emocional del profesor de matemáticas". Lima, Perú, agosto de 2017. 
La Figura 2 es una producción de una profesora de bachillerato que cursa una Maestría en Docencia de las Matemáticas ofertada en una Universidad de México. Ella dibujó una emoción positiva y una negativa de la clase de matemáticas; por lo expresivo de sus dibujos fue fácil identificar las emociones: la alegría de que los alumnos le presten atención y la tristeza cuando esto no ocurre.

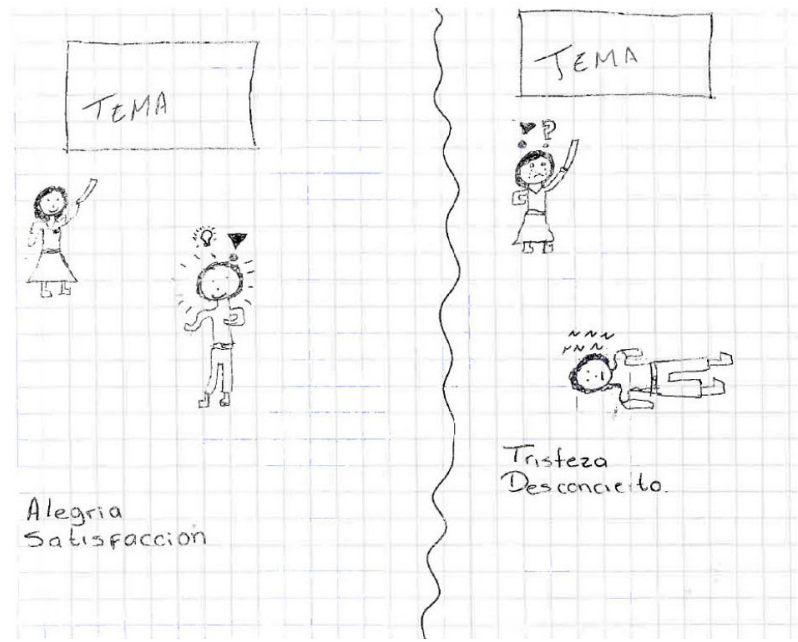

Figura 2. Emociones positivas y negativas en la clase de matemáticas.

Fuente: elaboración propia

Centremos la atención en la Figura 2 y obsérvese cómo la docente, el tema y el estudiante se ven involucrados en la situación desencadenante de la emoción. A la izquierda aparece la docente con una sonrisa, debido a que el alumno ha entendido su explicación del tema, esto se deduce al observar al alumno con un foco sobre la cabeza, lo que da cuenta de una emoción positiva que ella etiqueta como alegría y satisfacción. Al lado derecho, aparece una situación opuesta: la docente aparece con una mueca triste, con lágrimas, con signos de admiración e interrogación sobre su cabeza. Consideramos que con todos estos signos ella expresa su desconcierto ante la situación. En el caso del alumno, la serie de zetas junto a él, nos lleva a pensar en el aburrimiento y la falta de atención. Con esta última imagen, la docente representa la tristeza y el desconcierto cuando el estudiante no entiende el tema que ella explica. Interpretamos que la situación que desencadena la emoción, positiva y la negativa, es el aprendizaje de los temas. 
Como se describió antes, a la profesora se le facilita identificar la emoción que experimenta y la situación que la desencadena. Cuando mostró su dibujo en clase, todos logramos identificar la emoción que quería comunicar. Esta es una habilidad que no todos tenemos desarrollada, de ahí la importancia del ejercicio que nos ayuda a poder nombrar eso que sentimos.

\section{LA NARRATIVA}

Los humanos son, por naturaleza narradores de historias (Connelly y Clandinin, 1990) y relatos, y eso ocurre en todas las culturas. Su objeto suele ser variado y, entre otros aspectos, permiten entretener, educar o conocer aspectos ligados con experiencias vividas. En el caso de las narraciones, variados personajes, argumentos, gestos y expresiones forman parte de ellas, por eso se les considera relevantes y valiosas para cuando se desea conocer realidades que dependen de la manera como los sujetos las viven, visionan y perciben su mundo (Martínez-Padrón; 2011). Todo eso suscita multiplicidad de miradas y puntos de vista que le dan importancia a la investigación narrativa en vista de que "aporta la posibilidad de aproximarse a vivencias sociales desde relatos individuales y también, a la resignificación subjetiva de la realidad a propósito de los cambios de ésta mientras se narra a lo largo del tiempo" (Arias y Alvarado, 2015, p. 178). La narrativa, por tanto, permite estudiar maneras y formas en que los humanos experimentan el mundo.

En el campo de la Matemática Educativa, las narraciones se usan cada vez con mayor frecuencia, especialmente en investigaciones sobre cuestiones afectivas (Di Martino y Zan, 2010; Martínez-Sierra y García-González, 2014; 2016; 2017). Al implementar esta técnica en entornos escolares y al momento en que ellos hacen el papel de participantes de algún curso, usualmente se pide a los docentes que se tomen un tiempo libre, fuera de actividades cotidianas, para escribir su historia como docentes de matemáticas, posteriormente cada uno la lee en clase y quien escucha debe de comentar acerca de una experiencia positiva o negativa del relato. Esto, además, es un buen ejercicio para reflexionar sobre la práctica educativa, solo que a diferencia del dibujo requiere de tiempo para escribir, escuchar y ser escuchado.

Enseguida se exponen algunos fragmentos de narraciones de cuatro profesores de distintos niveles educativos, donde el objetivo es que el lector se percate 
de la parte emocional narrada. Para efectos de identificación de esos profesores, los hemos distinguido con la siguiente nomenclatura: $P_{i}$, donde $i=A, B, C$ y $D$.

- $P_{\mathrm{A}}$ : Profesor de nivel secundaria que atiende a estudiantes de 12-15 años, y tiene 8 años de experiencia docente.

- $P_{\mathrm{B}}$ : Profesor de nivel primaria que atiende a estudiantes de 6-12 años, y tiene 12 años de experiencia docente.

- $P_{C}$ : Profesor de nivel bachillerato que atiende a estudiantes de 15-18 años, y posee 8 años de experiencia docente.

- PD: Profesor de nivel secundaria, atiende a estudiantes de 12-15 años, y posee 2 años de experiencia docente.

También se establece que en cada evidencia narrativa, la palabra que concreta la expresión emocional se destaca en cursivas y la situación desencadenante se subraya.

El primero de estos profesores es $\mathrm{P}_{\mathrm{A}^{\prime}}$ quien manifiesta emociones de insatisfacción y descontento desencadenadas por el incumplimiento de tareas por parte de los estudiantes. Para él es importante que las tareas sean entregadas a tiempo, porque quiere inculcar el valor de la puntualidad. Sin embargo, esta forma de proceder fue vista por algunos padres de familia como reflejo de un mal profesor, situación que lo obligó a volverse más tolerante con la entrega de tareas, aunque ocasiona que no disfrute sus clases.

$\mathrm{P}_{\mathrm{A}}$ : Hasta el momento no me siento satisfecho conmigo, no me siento feliz dando clases, ya que observo que si pido las tareas en tiempo y forma los padres en vez de que me lo agradezcan, me tachan de mal profesor, he observado que a la mayoría de los padres lo que les importa es la calificación, y no que sus hijos aprendan. Esta situación me obligó a ser más flexible en la revisión de tareas, actividades, etc., cosa que no me gusta.

Pero también reconoce emociones positivas, como el orgullo al ver que sus estudiantes continuaron su educación escolar y se encuentran ya cursando una carrera profesional, y aún más, se siente satisfecho cuando ellos reconocen el papel que ha jugado para que se encuentren ahí. 
$\mathrm{P}_{\mathrm{A}}$ : Me hace sentir orgulloso de mi trabajo cuando encuentro a mis ex-alumnos estudiando sus carreras, me saludan y me dan las gracias por lo que les he enseñado, esto me da una satisfacción enorme, porque reconocen mi trabajo.

El segundo caso involucra a $\mathrm{P}_{\mathrm{B}}$, un profesor con un amplio conocimiento emocional, quien reconoce que la relación afectiva que tiene con sus estudiantes ha favorecido la relación profesor-estudiante en el aula de clases, y comenta sobre el aprecio que sus estudiantes le demuestran, mismo que es correspondido por él. Creemos que esta situación la favorece la personalidad del profesor y la edad de los estudiantes, ya que en los primeros años escolares, éstos tienden a ser más afectivos y sociables, con el paso de los años este comportamiento va cambiando.

$\mathrm{P}_{\mathrm{B}}$ : .... cierro con el fruto más dulce y que más he saboreado: el aprecio de mis estudiantes. Este hecho siempre lo he tomado como la fuente que me alienta y me empuja para ser mejor cada día. Esta estrecha relación afectiva que logro establecer con la mayoría de mis estudiantes desde un inicio es un componente positivo de mi práctica docente. Ellos comúnmente expresan de manera abierta sus sentimientos hacia mí y les gusta mucho que les dé un abrazo. En la hora del recreo no quieren despegarse de mí, quieren seguir platicando, pero no sobre lo que estudiamos en el aula, sino de nuestras vidas.

El tercer profesor es $P_{C}$ un docente de nivel bachillerato que se siente contento por la labor que realiza, sorteando las malas prácticas de sus estudiantes. En su discurso se da evidencia de la tolerancia que practica en la clase de matemáticas, particularmente en los cursos de Cálculo, a los que en la mayoría de los casos los alumnos llegan con conocimientos muy escasos de Aritmética y Álgebra que, como se sabe, son necesarios para el estudio y comprensión del Cálculo. Comenta que en su institución es muy común que algunos profesores de matemáticas no impartan sus cursos y dejen a los estudiantes sin clases, fomentando así malas prácticas en los últimos. Él lucha contra estas malas prácticas, asiste con puntualidad a sus cursos, no se muestra autoritario con los alumnos, pero si se hace respetar, los escucha y responde a sus preguntas. Este comportamiento ha desencadenado en una relación de confianza de sus alumnos.

$\mathrm{P}_{\mathrm{c}}$ : Trato de combatir con hechos las malas prácticas que por costumbres se han hecho hábitos, tanto en profesores como en alumnos. Trato de estar siempre puntual 
en el aula, preparo mi clase, no me impongo a los estudiantes, los escucho, no me molesta que me pregunten lo mismo y repetir la explicación del tema cuantas veces sea necesaria, creo que eso les ha generado un cierto grado de confianza en mí... Esta situación me hace sentir bien con mi trabajo.

El último de los casos es $\mathrm{P}_{\mathrm{D}}$, un profesor novel de nivel secundaria cuyo conocimiento emocional lo llevó a modificar su práctica docente. Este profesor no tiene formación docente, pero si el perfil para impartir matemáticas, es arquitecto. Su caso es ejemplar y digno de ser mencionado. Al aceptar trabajar como docente de matemáticas se dio cuenta que las matemáticas que conocía no eran suficientes para impartir clases, esta situación le desencadenaba emociones negativas. En cada clase que desarrollaba, sentía temor, agobio y estrés, signos claros de ansiedad matemática.

$\mathrm{P}_{\mathrm{D}}$ : Cuando inicié en segundo año de secundaria como mi conocimiento de la matemática era muy poco me sentía frustrado por no poder enseñar más...En mi caso el desconocimiento de algo me da temor y ese temor me agobiaba, ese agobio creo que se notaba en la clase con mis alumnos porque estaba molesto, irritable, no me concentraba.

Ser consciente de su exiguo conocimiento de la matemática y de las emociones que esto le ocasionaba, llevó a $\mathrm{P}_{\mathrm{D}}$ a buscar ayuda para mejorar como docente, y decidió ingresar a una Maestría en Docencia de la Matemática. Al estar cursándola, recibió apoyo de sus profesores y compañeros, además de un proceso de acompañamiento para regular sus emociones. En particular, el acompañamiento lo llevó a modificar su práctica docente y al reforzar sus conocimientos matemáticos adquirió seguridad para conducir sus clases (García-González, y Martínez-Sierra, 2018).

$\mathrm{P}_{\mathrm{D}}$ : Yo creía que ser maestro de matemáticas era muy difícil y agobiante, hasta llegué a pensar que yo no servía para eso, pero ahora sé que se puede llegar a disfrutar ser profesor... yo busqué prepararme más en el tema, me propuse estudiar una Maestría en Docencia de la Matemática que me dio las herramientas y la seguridad que requería para desempeñarme en el ámbito de la educación matemática. 
El caso de ansiedad matemática de $P_{D}$ puede ser representativo de profesores noveles, quienes, aunque comprometidos con su labor, en sus primeros años experimentan emociones negativas, mismas que superan con el paso de los años (Anttila, Pyhältö, Soini y Pietarinen, 2016). Este caso resulta especial por dos razones, él no tenía la formación para ser docente de matemáticas y se inscribió en una Maestría en Docencia de la Matemática para convertirse en profesor de matemáticas. De su historia podemos aprender que la ansiedad matemática se puede aliviar cuando hay un reconocimiento explícito de quien la padece y disposición para hacerlo.

Resumiendo lo encontrado, podemos observar que la teoría OCC y la perspectiva fenomenológica resultan útiles para conocer y describir las emociones, y las situaciones que las desencadenan mediante los dibujos y las narraciones dadas por los profesores en estudio. Valoraciones como las declaradas por $P_{D}$ al momento de reconocer sus falencias docente de matemáticas, evidencian la presencia de factores éticos, morales, sociales, y psicológicos que emergieron al evaluar su propio conocimiento profesional como docente de matemáticas, habida cuenta de sentirse frustrado, temeroso y hasta agobiado por la valoración de no poder enseñar más, situación ética que marcó el hecho de tomar la decisión de prepararse mejor en matemáticas, a fin de controlar las emociones que le causaban irritabilidad y hasta falta de concentración. Situaciones como ésta se enmarcan en el contexto sociocultural de la clase que también se evidencia en el caso de $\mathrm{P}_{\mathrm{A}}$, aunque ahora se concreta con una tendencia conductual de los estudiantes al no hacer efectiva la entrega de tareas en el tiempo acordado. Asuntos de esta naturaleza producen insatisfacciones que decantan en descontentos sociales, en este caso, por algunos padres de los estudiantes quienes emiten opiniones que causan malestar de orden psicológico y hasta agitaciones físicas que pueden delatarlo ante otros miembros de la clase. Incluso, pueden afectar el juicio del profesor, si decidiera no ser tolerante ante estas situaciones asociadas con su estado de ánimo.

En ambos casos, se marca un referente cultural que obliga a regular el comportamiento y las acciones de los sujetos interactuantes en la dinámica de la clase, de manera que las respuestas emocionales pueden girar en muchas direcciones, según la experiencia emocional que posean los protagonistas de la clase. En relación con el afecto, se puede notar que la situación narrada por $\mathrm{P}_{\mathrm{B}}$ es grata para él y para sus estudiantes por el hecho de estar cargada de emociones positivas. Eso se acopla a un referente psicológico 
detectado en la personalidad del profesor y la de sus estudiantes. Todo eso genera un proceso de valoración favorable y manifiesto en la satisfacción de ser cada día mejor.

\section{A MANERA DE CIERRE}

La pretensión de este escrito ha sido la comunicación de resultados de investigación sobre las emociones de docentes, desde el área de la Matemática Educativa. Se han mostrado las emociones más frecuentes que emergen en las clases de matemáticas y lo que las desencadenan, guiados siempre por la teoría OCC y por las vivencias de los participantes conocidas a través de dos técnicas que pueden ayudar al profesor a alfabetizarse sobre su conocimiento emocional, lo que implica el reconocimiento de las emociones que se experimentan en clase y las situaciones que las desencadenan. Desde esa experiencia se puede concluir que el conocimiento emocional permite:

1. Regular nuestras emociones, una vez que somos consciente de ellas, baste como ejemplo el caso del profesor $\mathrm{P}_{\mathrm{D}}$, quien padecía de ansiedad matemática debido a su poco conocimiento matemático, una vez que lo fortaleció, ganó seguridad, misma que se manifestó en sus clases de secundaria y en la relación con sus estudiantes.

2. Ayudar a los estudiantes a conocer sus propias emociones, explicarles que es sano experimentar emociones en el salón de clases, porque es algo natural en los seres humanos. Desde allí, podemos asistirlos a que ellos le den un nombre a eso que sienten, a separar las emociones negativas de las positivas y ayudarlos a reconocer qué puede desencadenar cada tipo de emociones, y las acciones que en consecuencia se deben tomar para tener una sana vida emocional. A futuro, esto fomentará la formación de seres capaces de tomar decisiones acertadas en su vida, con una conciencia plena de sí mismos.

En relación con lo metodológico se hace necesario resaltar las bondades del paradigma fenomenológico interpretativo, puesto que permitió hurgar en la interioridad de los profesores de matemáticas que formaron parte del grupo de estudio. Desde ese lugar epistemológico y con apoyo de lo dibujado y narrado por ellos, se pudo dar cuenta de sus experiencias, visiones y percepciones 
aderezadas por sus contextos particulares de clases, teniéndose la seguridad de que de todos esos insumos, cargados de significados, emergieron de la realidad donde se encuentran inmersos.

Tenemos claro que estudiar las emociones, su naturaleza y las situaciones que las desencadenan siempre será una tarea exigente y, por ende, su conocimiento profundo no siempre puede ser develado con la debida finura. Manejar los estados emocionales tampoco es una tarea fácil, máxime cuando estamos claros de las dificultades que existen para dominarlas o controlarlas, debido a que pueden secuestrar hasta la razón de los sujetos. Por tanto, consideramos que quedan muchas conjeturas abiertas a solicitud de su propia esencia, en tal sentido, recomendamos; (a) ahondar en trabajos donde la línea fuerte sean los factores emocionales intrínsecos ligados con las matemáticas, penetrando, con rigor, en los fenómenos y reacciones relacionadas con la mente y el cuerpo, en virtud de su carácter complejo y difuso; y (b) atender la construcción y aplicación de instrumentos que puedan soterrar y medir, desde diferentes vértices y miradas epistemológicas, aspectos como la ansiedad matemática y la aversión hacia la matemática, en vista de que quedan muchos velos por quitar, sobre todo en lo referido a la aversión que, como se sabe, debe ser atendida con urgencia, habida cuenta de conocer la presencia de un importante contingente de estudiantes que rechazan y odian a las matemáticas y suelen trastornar la actuación de los docentes que las enseñan (García-González y Martínez-Sierra, 2018). Calle (2002) advierte que además de perturbar la mente, la aversión "es una actitud...de rechazo, resistencia, conflicto, oposición y acritud, que puede derivar en animadversión, odio, ira, malevolencia, afán de venganza, irritabilidad crónica, agresividad e incluso violencia" (p. 59). Todo esto puede colocar a los estudiantes en condición de irascibles, lo cual representa una situación angustiante y compleja a la que tiene que arrostrar el docente de matemáticas.

\section{REFERENCIAS}

Aguilar, M., Rosas, A., Zavaleta, J. y Romo-Vázquez, A. (2016). Exploring high-achieving students' images of mathematicians. International Journal of Science and Mathematics Education, 14, 527-548.

Albrecht, K. (2006). Inteligencia social, (G. Dols, Trad.). Javier Vergara Editor (Trabajo original publicado en 2006). 
Anttila, H., Pyhältö, K., Soini, T. y Pietarinen, P. (2016). How does it feel to become a teacher? Emotions in Teacher Education. Social Psychology of Education, 19(3), 451-473.

Arias Cardona, A. y Alvarado Salgado, S. (2015). Investigación narrativa: apuesta metodológica para la construcción social de conocimientos científicos. CES Psicología, 8(2), 171-181.

Bekdemir, M. (2010). The pre-service teachers' mathematics anxiety related to depth of negative experiences in mathematics classroom while they were students. Educational Studies in Mathematics, 75(3), 311-328. http://doi.org/10.1007/s10649-010-9260-7.

Calle, R. (2002). Las zonas erróneas de tu mente. Ediciones Temas de Hoy S. A.

Connelly, M. y Clandinin, J. (1990). "Stories of experience and narrative inquiry". Educational Researcher, 19(5), 2-14.

Coppola, C., Di Martino, P., Pacelli, T. y Sabena, C. (2012). "Primary teachers' affect: A crucial variable in the teaching of mathematics". Nordic Studies in Mathematics Education, 17, 3-4.

Damasio, A. (2009). En busca de Spinoza. Neurobiología de la emoción y los sentimientos. (J. Ros, Trad.), Editorial Crítica S. L.

De Bellis, V. y Goldin, G. (2006). Affect and meta-affect in mathematical problem solving: a representational perspective. Educational Studies in Mathematics, 63, 131-147.

Di Martino, P. y Sabena, C. (2011). "Elementary pre-service teachers' emotions: shadows from the past to the future". In K. Kislenko (Ed.), Current state of research on mathematical beliefs XVI (pp.89-105). Tallinn University.

Di Martino, P. y Zan, R. (2010). "Me and maths: towards a definition of attitude grounded on students' narratives". Journal of Mathematics Teacher Education, 13(1), 27-48.

Di Martino, P. y Zan, R. (2011). "Attitude towards mathematics: a bridge between beliefs and emotions". ZDM- The International Journal on Mathematics Education, 43(4), 471-482.

Fennema, E., y Sherman, J. A. (1976). Fennema-Sherman mathematics attitude scales: Instruments designed t-o measure attitudes toward the learning of mathematics by females and males. Journal for Research in Mathematics Education, 7(5), 324-326.

Font, V. (2011). Investigación en didáctica de las matemáticas en la educación secundaria obligatoria. En M. Marín, G. García, L.J. Blanco y M. Medina (Eds.), Investigación en Educación Matemática XV, (pp. 165-194). Sociedad Española de Investigación en Educación Matemática y Servicio de publicaciones de la Universidad de Castilla-La Mancha.

García-González, M. y Martínez-Sierra, G. (2016). “Emociones en profesores de matemáticas: un estudio exploratorio". En J.A Macías, A. Jiménez, J. L. González, M. T Sánchez, 
P. Hernández, C. Fernández, F.J Ruiz, T. Fernández y A. Berciano (Eds.), Investigación en Educación Matemática XX (pp. 247-252). SEIEM.

García-González \& Pascual-Martín (2017). De la congoja a la satisfacción: el conocimiento emocional del profesor de matemáticas. IE Revista de Investigación Educativa de la Rediech, 8(15), 133-148.

García-González, M. S. y Martínez-Sierra, G. (2018). Diego: Una historia de superación de ansiedad matemática en profesores. En L. J. Rodríguez-Muñiz, L. Muñiz-Rodríguez, A. Aguilar-González, P. Alonso, F. J. García García y A. Bruno (Eds.), Investigación en Educación Matemática XXII (pp. 221-230). SEIEM.

Gaxiola, P. (2005). La inteligencia emocional en el aula. Segunda edición. Aula nueva. SM.

Goldin, G., Hannula, M., Heyd-Metzuyanim, E., Jansen, A., Kaasila, R., Lutovac, S., Di Martino, P., Morselli, F., Middleton, J., Pantziara, M. y Zhang, Q. (Eds.). (2016). Attitudes, Beliefs, Motivation and Identity in Mathematics Education. An Overview of the Field and Future Directions. Springer.

Goleman, D. (1996). La inteligencia emocional, (E. Mateo, Trad.). España: Javier Vergara Editor (Trabajo original publicado en 1995).

Goleman, D. (2006). Inteligencia social. La nueva ciencia de las relaciones humanas. Editorial Kairós.

Gómez, R., Blanco, L., Cárdenas, J. y Guerrero, E. (2012). Desencadenantes del estrés y emociones en docentes de matemáticas de secundaria. Estudio realizado con una escala de elaboración propia. En V. Mellado, L. Blanco, A. Borrachero y J. Cárdenas (Eds), Las emociones en la enseñanza y el aprendizaje de las ciencias y las matemáticas, volumen I; (pp.45-66), Grupo de Investigación DEPROFE.

Hannula, M. S., Liljedahl, P., Kaasila, R., y Rösken, B. (2007). Researching relief of mathematics anxiety among pre-service elementary school teachers. In J.H. Woo, H.C. Lew, K.S. P. Park, y D.Y. Seo (Eds.), Proceedings of 31st Annual Conference for the Psychology of Mathematics Education, vol. 1 (pp. 153-156). Seoul, Korea.

Henrion, C. (1997). Women in Mathematics: The addition of difference. University Press, Bloomington and Indianapolis.

López, C. y Valls, C. (2013). Coaching educativo, las emociones al servicio del aprendizaje. SM.

Maaß, J. y Schlöglmann, W. (2009). Beliefs and attitudes in Mathematics Education. New Research Results. Sense Publishers Totterdam.

Mager, R. (1984). Developing attitude toward learning. Kogan.

Martínez- Miguélez, M. (2006). Ciencia y arte en la metodología cualitativa (2ed.). Trillas. 
Martínez-Padrón, O. J. (2008). Actitudes hacia la Matemática. Sapiens, 9(2), 237-256.

Martínez-Padrón, O. J. (2016). ¿Qué dicen los docentes paraguayos en cuanto al afecto en el aprendizaje de la Matemática?: Una mirada desde el Curso Ñanduti. UNIÓN, 45, 24-43.

Martínez-Padrón, O. J. (2011). El afecto en el aprendizaje de la Matemática, Texto de Curso Nanduti: Paraguay, Curso Iberoamericano de Formación Permanente de Profesores de Matemática. Centro de Altos Estudios Universitarios. Organización de Estados Iberoamericanos para la Educación, la Ciencia y la Cultura (OEI). Andalucía, España.

Martínez-Padrón, O. J., Contarino, A. y Ávila, J. (2015). Aspectos emocionales que impactan el desempeño de los estudiantes en el aula de Matemática. En R. Flores (Ed.), Acta Latinoamericana de Matemática Educativa 28, 181-189. Comité Latinoamericano de Matemática Educativa A. C.

Martínez-Sierra, G. y García-González, M. (2014). “High school students' emotional experiences in mathematics classes". Research in Mathematics Education 16(3), 234-250.

Martínez-Sierra, G. y García-González, M. (2016). “Undergraduate mathematics students' Emotional experiences in linear algebra". Educational Studies in Mathematics 91(1), 87-106.

Martínez-Sierra, G. \& García-González, M.S. (2017). Students' emotions in the high school mathematics classroom: The appraisals in terms of a structure of goals. International Journal of Science and Mathematics Education 15(2), 349-369. DOI: 10.1007/ s10763-015-9698-2.

McLeod, D. B., y Adams, V. M. (Eds.) (1989). Affect and mathematical problem solving: A new perspective. Springer Verlag.

Ortony, A., Clore, G. L., y Collins, A. (1996). The cognitive structure of emotions. (J. Martínez y R. Mayoral, traductores). España: Siglo XXI. (Trabajo original publicado en 1988).

Philipp, R. (2007). "Mathematics teachers' beliefs and affect". In Frank Lester (Ed.), Handbook of research on mathematics teaching and learning (pp. 257-315). Information Age Publishing.

Picker, S. y Berry, J. (2000). "Investigating pupils' images of mathematicians". Educational Studies in Mathematics, 43(1), 65-94.

Polya, G. (1965). Cómo plantear y resolver problemas (J. Zagazagoitía, Trad). Editorial Trillas.

Rodríguez, J., Guevara, A. y Viramontes, A. (2017). Síndrome de burnout en docentes. IE Revista de Investigación Educativa de la Rediech, 7(14), 45-67. 
Schoenfeld, A. (1983). Episodes and executive decisions in mathematical problem-solving skills. En R. Lesh y M. Landau (Eds.), Acquisition of mathematical concepts and processes (pp. 345-395). Academic Press.

Schutz, P., y Zembylas, M. (2009). Introduction to advances in teacher emotion research: The impact on teachers lives. En P. Schutz, M. Zembylas (Eds.), Advances in teacher emotion research: The impact on teachers lives. Springer.

Solomon, R. (2008). The philosophy of emotions, In M. Lewis, J. Haviland-Jones, y L. Feldman (Eds), Handbook of emotions, 3rd ed, (pp. 3-16). The Guilforf Press.

Stets, J., y Turner, J. (2008). The sociology of emotions, In M. Lewis, J. Haviland-Jones, y L. Feldman (Eds), Handbook of emotions, 3rd ed, (pp. 32-46). The Guilforf Press.

Ursini, S. y Sánchez, G. (2008). "Gender, technology and attitude towards mathematics: a comparative longitudinal study with mexican students". ZDM- The International Journal on Mathematics Education, 40(4), 559-577.

Yazlik, D. y Erdogan, A. (2017). "Image of the high school students towards mathematicians". Journal of educational and instructional studies in the world, 6(4), 1-14.

María S. García GonZÁleZ

Dirección: Facultad de Matemáticas. Universidad Autónoma de Guerrero, México; mgargonza@gmail.com

Teléfono: $\quad 01$ (747) 4719310 Ext. 4138 y 4137. 